\title{
Editorial
}

\section{Defects by design: Quantum nanophotonics in emerging materials}

\author{
https://doi.org/10.1515/nanoph-2019-0437
}

Point defects in wide-bandgap semiconductors have emerged as leading platforms for quantum information science and nanophotonics, but most research has concentrated on only a few defect systems and host materials. Building on recent success in harnessing select defects like the diamond nitrogen-vacancy center for quantum computing, quantum optics, magnetometry, and biosensing, an opportunity exists to identify systems in new materials that are optimized to improve the performance of applications like these, or that can enable entirely new avenues of research. Given the vast number of potential materials and defect systems, however, it remains a major challenge to theoretically predict and experimentally identify promising candidates in a systematic way. This special issue surveys the current understanding of optically-active semiconductor defects, highlighting new directions for research that harness the unique properties afforded by emerging materials.

In October 2018, we organized an incubator meeting together with the Optical Society, which brought together scientists from around the world to discuss the following questions:

\section{Why defects?}

- What makes a "good" defect for quantum science and technology?

- What application areas are best suited to quantum defects, and which are not?

- What new areas are ripe for exploration using quantum defects?

\section{Defects in new materials}

- How can we efficiently create and identify new defects?

- How can we control defect creation and placement at the atomic scale?

- What opportunities are provided by host materials beyond group IV?

\section{Defects by design}

- How can we predict defect properties to guide discovery?

- What new theoretical tools are needed to give a holistic treatment and prevent "convergence to experiments"?

- What is the importance and influence of the material host (e.g. dimensionality, composition, symmetry)? What are the most promising materials?

- How can we engineer desired defect properties (e.g. using nanophotonics, materials properties, device design, or active control schemes)?

The vibrant discussions at the incubator meeting emphasized the need for more research on these themes, and we subsequently organized this collection of research, review, and perspective articles to facilitate in-depth explorations and dissemination of related ideas. The articles in this special issue broadly address the key themes of the incubator.

In a perspective in this special issue [1], we consider the exciting opportunities for basic science and new applications based on defects in new materials. Taking a historical view, we emphasize the key properties of the predominant materials and defect systems - especially the nitrogen-vacancy (NV) center in diamond - that have propelled the existing research field. We propose that it should be possible to identify new defects with properties that are optimized for particular research areas and applications, through a search guided by judicious combinations of heuristics, predictions, and experiments. We survey the primary challenges related to theoretical prediction, materials growth and processing, and experimental characterization, highlighting the need for advances in all three areas.

The historical perspective on well-established quantum defects is elaborated through reviews by Smith et al. [2] and Gali [3], who, respectively, discuss the existing methodologies for creating NV centers in diamond, and for predicting 
their quantum properties using ab initio theory. Smith et al. emphasize the relative advantages and disadvantages of different synthesis methods, where application requirements drive specific choices to balance the tradeoffs. The diversity of approaches highlights the opportunity for continued research on this topic and for the adaptation of similar methods to create quantum defects in other materials. Gali's comprehensive review of $a b$ initio theory as applied to the diamond NV center covers older, well-established approaches alongside many recent advances. Ultimately, our success in predicting and understanding new quantum defects from the theoretical standpoint will depend on the accuracy of our theoretical methodologies. As the most well-characterized quantum defect, the NV center is an obvious testbed for such developments. To this end, Gail points out the success of $a b$ initio theory in explaining many key features, while highlighting some questions that are still hotly debated.

The diamond NV center remains at the forefront of research with quantum defects for good reasons. Levine et al. [4] review the state-of-the-art in wide-field magnetic imaging using NV centers in the quantum diamond microscope. Their article is a detailed guide for the design and operation of such microscopes, which have applications in biology, materials science, and geoscience. Meanwhile, Oberg et al. [5] present an intriguing theoretical proposal to transfer an electron with a quantum-coherent spin state from one NV center to another one in a diamond quantum wire via stimulated Raman adiabatic passage. This method could lead to a scalable architecture for quantum computers based on NV centers or other quantum defects.

Amazingly, despite decades of research, some fundamental properties of the diamond NV center remain unknown. One of those mysteries is the fine structure of the NV center's neutral charge state. To address this question, Barson et al. [6] present a magnetic circular dichroism study, where the fine structure of the neutral NV center has been determined. They describe a spin-orbit Hamiltonian consistent with the data, and discuss possible reasons why the structure was not observed before.

The bulk of the issue is devoted to articles that discuss the state of the art in identifying and engineering new quantum defects. Balasubramanian et al. [7] report the occurrence of the so-called ST1 center in natural diamond. This center has previously been observed only in synthetic diamond, and its origin is unknown. The authors analyze the ST1 center's spectral characteristics and proffer ideas regarding its atomistic structure.

Zhong and Goldner [8] review progress and challenges in realizing nanoscale and low-dimensional devices based on solids doped with rare-earth ions. Research in this field has been propelled by the long coherence times and high optical quantum efficiencies of rare-earth ions in bulk crystals, and nanophotonic devices based on these systems may enable scalability and complementary device functionality.

Several articles describe advances in understanding and control over quantum defects in two-dimensional (2D) semiconductors, which have emerged in recent years as promising host materials with unique physical, electronic, and optical properties. The 2D geometry enables facile device integration, and it is ideally suited for quantum sensing if defects with suitable quantum properties can be identified and controlled. Chakraborty et al. [9] review the current understanding regarding the nature of quantum light emission in this rapidly advancing sub-area, including mechanisms driven by defects, strain, or Moire lattice potentials. Lee and Gupta [10] highlight the exciting opportunities and key challenges for characterizing, creating, and controlling point defects using scanned probe techniques, paying special attention to the unique potential of 2D materials and the need to develop methodologies that integrate optical and magnetic resonance techniques.

Working with hexagonal boron nitride (h-BN), where visible-wavelength quantum emitters are attributed to as-yetunknown point defects, Bommer and Becher [11] study the emitters' spectral, temporal, and polarization properties; on the basis of these measurements they propose an intriguing double-defect model in which each emitter is characterized by two independent electronic transitions. In related work, Li et al. [12] report two post-transfer treatments that generate quantum emitters with high purity and narrow room-temperature linewidths in films of chemical-vapor-depositiongrown h-BN. These key features are necessary for the use of h-BN's quantum emitters in photonic devices, but they have generally been lacking in samples contaminated by the transfer process. Finally, Proscia et al. [13] engineer devices based on ensembles of quantum emitters in h-BN films coupled to surface plasmon resonances in an underlying array of silver nanopillars, demonstrating the ability to tune both the intensity and direction of the emission.

Ultimately, this collection of articles reflects the diversity of questions, methodologies, and applications that characterize research with quantum defects. We are grateful to all the authors who have contributed their work and ideas, and we hope this is a useful resource to anyone who wishes to learn about this fascinating and rapidly-expanding topic. 
Acknowledgment: We are grateful to the Optical Society for hosting the 2018 incubator meeting on this topic, which was also supported by the National Science Foundation, the Department of Energy, the Air Force Office of Scientific Research, the Army Research Office, and Element Six.

\section{References}

[1] Bassett LC, Alkauskas A, Exarhos AL, Fu K-MC. Quantum defects by design. Nanophotonics 2019;8:1867-88.

[2] Smith JM, Meynell SA, Bleszynski Jayich AC, Meijer J. Colour centre generation in diamond for quantum technologies. Nanophotonics 2019;8:1889-906.

[3] Gali Á. Ab initio theory of the nitrogen-vacancy center in diamond. Nanophotonics 2019;8:1907-43.

[4] Levine EV, Turner MJ, Kehayias P, et al. Principles and techniques of the quantum diamond microscope. Nanophotonics 2019;8: 1945-73.

[5] Oberg LM, Huang E, Reddy PM, et al. Spin coherent quantum transport of electrons between defects in diamond. Nanophotonics 2019;8:1975-84.

[6] Barson MS], Krausz E, Manson NB, Doherty MW. The fine structure of the neutral nitrogen-vacancy center in diamond. Nanophotonics 2019;8:1985-91.

[7] Balasubramanian P, Metsch MH, Reddy P, et al. Discovery of ST1 centers in natural diamond. Nanophotonics 2019;8:1993-2002.

[8] Zhong T, Goldner P. Emerging rare-earth doped material platforms for quantum nanophotonics. Nanophotonics 2019;8:2003-15.

[9] Chakraborty C, Vamivakas N, Englund D. Advances in quantum light emission from 2D materials. Nanophotonics 2019;8:2017-32.

[10] Lee D, Gupta JA. Perspectives on deterministic control of quantum point defects by scanned probes. Nanophotonics 2019;8:2033-40.

[11] Bommer A, Becher C. New insights into nonclassical light emission from defects in multi-layer hexagonal boron nitride. Nanophotonics 2019;8:2041-8.

[12] Li C, Xu Z-Q, Mendelson N, Kianinia M, Toth M, Aharonovich I. Purification of single-photon emission from hBN using post-processing treatments. Nanophotonics 2019;8:2049-55.

[13] Proscia NV, Collison RJ, Meriles CA, Menon VM. Coupling of deterministically activated quantum emitters in hexagonal boron nitride to plasmonic surface lattice resonances. Nanophotonics 2019;8:2057-64.

\section{Audrius Alkauskas}

Center for Physical Sciences and Technology (FTMC), Vilnius LT-10257, Lithuania, e-mail: audrius.alkauskas@ftmc.lt. https://orcid.org/0000-0002-4228-6612

\section{Lee C. Bassett}

Department of Electrical and Systems Engineering, University of Pennsylvania, Philadelphia, PA 19104, USA, e-mail: lbassett@seas.upenn.edu. https://orcid.org/0000-0001-8729-1530

\section{Annemarie L. Exarhos}

Department of Physics, Lafayette College, Easton, PA 18042, USA, e-mail: exarhosa@lafayette.edu. https://orcid.org/0000-0003-3026-7737

\section{Kai-Mei C. Fu}

Department of Physics and Department of Electrical and Computer Engineering, University of Washington, Seattle, WA 98195, USA, e-mail: kaimeifu@uw.edu. https://orcid.org/0000-0003-4775-8524 